\title{
Expanding the phenotypic spectrum of MBOAT7-related intellectual disability
}

\author{
Joseph E. Jacher ${ }^{1}$ ( ) | Nikita Roy ${ }^{2}$ | Mohammad Ghaziuddin ${ }^{2}$ | Jeffrey W. Innis ${ }^{1,3,4}$
}

\author{
${ }^{1}$ Department of Pediatrics and Communicable \\ Diseases, Division of Pediatric Genetics, \\ Metabolism and Genomic Medicine, University \\ of Michigan, Ann Arbor, Michigan \\ ${ }^{2}$ Psychiatry, University of Michigan, Ann \\ Arbor, Michigan \\ ${ }^{3}$ Human Genetics, University of Michigan, Ann \\ Arbor, Michigan \\ ${ }^{4}$ Internal Medicine, University of Michigan, \\ Ann Arbor, Michigan \\ Correspondence \\ Jeffrey W. Innis, MD, PhD, D5240 Medical \\ Professional Building, 1500 E Medical Center \\ Drive, Ann Arbor, MI 48109. \\ Email: innis@med.umich.edu
}

\begin{abstract}
MBOAT7 gene pathogenic variants are a newly discovered and rare cause for intellectual disability, autism spectrum disorder (ASD), seizures, truncal hypotonia, appendicular hypertonia, and below average head sizes (ranging from -1 to -3 standard deviations). There have been only 16 individuals previously reported who have MBOAT7-related intellectual disability, all of whom were younger than 10 years old and from consanguineous relationships. Thus, there is a lack of phenotypic information for adolescent and adult individuals with this disorder. Medical genetics and psychiatric evaluations in a 14year-old female patient with a history of global developmental delay, intellectual disability, overgrowth with macrocephaly, metrorrhagia, seizures, basal ganglia hyperintensities, nystagmus, strabismus with amblyopia, ASD, anxiety, attention deficit hyperactivity disorder (ADHD), aggressive outbursts, and hyperphagia included a karyotype, methylation polymerase chain reaction for Prader-Willi/Angelman syndrome, chromosome microarray, and whole exome sequencing (WES), ADOS2, and ADI-R. WES identified a homozygous, likely pathogenic variant in the MBOAT7 gene (c.855-2A>G). This is the oldest known patient with MBOAT7-related intellectual disability, whose unique features compared with previously described individuals include overgrowth with macrocephaly, metrorrhagia, ophthalmological abnormalities, basal ganglia hyperintensities, unspecified anxiety disorder, and ADHD; combined type; and hyperphagia with the absence of appendicular hypertonia and cortical atrophy. More individuals need to be identified in order to delineate the full clinical spectrum of this disorder.
\end{abstract}

\section{KEYWORDS}

intellectual disability, MBOAT7, whole exome sequencing

\section{1 | INTRODUCTION}

\section{1 | Background}

Genetic testing for neurodevelopmental disorders has been rapidly increasing over the last decade. New genetic disorders are being discovered at a rate not seen before, especially with the use of whole exome sequencing (WES) (The Deciphering Developmental Disorders Study, 2017). WES has become the new gold standard test for patients with undiagnosed genetic disorders with a diagnostic yield, after an initial workup, of around 36\% (Clark et al., 2018). Johansen et al. (2016) were the first group to discuss pathogenic variants in MBOAT7 (OMIM 606048) as a rare cause for human neurodevelopmental delays, autism spectrum disorder (ASD), and seizures in a total of 16 individuals. ASD is a well-described developmental disorder with both social and communication deficits in addition to stereotyped, ritualistic, and/or repetitive behaviors (Nazeer \& Ghaziuddin, 2012). Children who have intellectual disability, with or without MBOAT7 pathogenic variants, are known to have higher 
overall rates of various psychopathologies (Dykens, 2000), which can include disruptive disorders, mood disorders, and/or anxiety disorders (Emerson, 2003). In this article, we report a 14-year-old female with MBOAT7-related intellectual disability who has a history of global developmental delay, intellectual disability, overgrowth with macrocephaly, metrorrhagia, seizures, basal ganglia hyperintensities, nystagmus, strabismus with amblyopia, ASD, attention deficit hyperactivity disorder (ADHD), anxiety, irritable mood, perseverative behaviors, aggressive outbursts, and hyperphagia.

\section{2 | MATERIALS AND METHODS}

\section{1 | Ethical compliance}

Informed consent under the University of Michigan IRB protocol HUM00065360 was obtained from the family for this article in compliance with institutional and national ethics regulations.

\section{2 | Chromosome analysis}

Chromosome analysis was performed on 20 metaphase cells from peripheral blood at the 550 band level of resolution using the G-banding method.

\section{3 | Prader-Willi/Angelman syndrome methylation testing}

Southern blot methylation analysis was performed on DNA obtained from peripheral blood used the SNRPN probe and digestion with Not I and Xba I.

\section{4 | Chromosome microarray analysis}

The chromosome microarray was performed using the Illumina CytoSNP-850 K (GRCh37/hg19 genome assembly) platform on DNA extracted from peripheral blood.

\subsection{Whole exome sequencing (trio)}

WES was performed at Ambry Genetics Corp. Genomic deoxyribonucleic acid was isolated from whole blood. Proband and parental samples were prepared using the SeqCap EZ VCRome 2.0 (Roche NimbleGen) or the IDT xGen Exome Research Panel V1.0. Final quantified libraries were seeded onto an Illumina flow cell and sequenced using paired-end, 100 or 150 cycle chemistry on the Illumina HiSeq or NextSeq. Variants were confirmed by Sanger sequencing.

\section{3 | RESULTS}

\section{1 | Medical genetics evaluation}

The female patient was born without a history of in utero teratogenic exposure, but the pregnancy was complicated by vaginal bleeding at
2 months' gestation as well as decreased fetal movements beginning at 6 months' gestation. She was born via complicated delivery (arduous labor and faint fetal heart tones) to a 23 year old, G1P1 mother at 38-4/7 weeks' gestation. Apgar scores were 8 and 9 at 1 and $5 \mathrm{~min}$, respectively. There was no reported consanguinity, though the parents are from the same village in Lebanon. The birth weight was $2,835 \mathrm{~g}$ (18th percentile), the birth length was $55 \mathrm{~cm}$ (+3 standard deviations $[S D]$ ), and the head circumference was $34 \mathrm{~cm}$ (54th percentile). Our patient had hypotonia throughout infancy that resolved in childhood. She had four simple partial seizures within a 24-hr period at 17 months of age and was treated with an antiepileptic for 7 months before being weaned off with no seizure recurrence. Vision assessments at ages 7 months and 3 years have been remarkable for intermittent esotropia, nystagmus, and unilateral strabismus with amblyopia; she underwent laser surgery for the strabismus at 1 year of age.

The most recent brain magnetic resonance imaging (MRI) was performed at 3.5 years of age and revealed flare signal hyperintensities within both basal ganglia, which appeared stable compared with the prior study at age 14 months. The signal abnormalities in the dentate nuclei bilaterally and retrotrigonal white matter bilaterally appeared slightly decreased in size and slightly less conspicuous compared with prior MRI. There were also signal abnormalities in the subcortical white matter of the left precentral gyrus and the anterior aspect of the superior frontal gyrus on the left, which was similar to the prior MRI. There were no new lesions appreciated. Neurological and neuroradiological evaluation of her head MRI revealed primarily cerebellar and basal ganglia involvement, regions known to be more susceptible to hypoxic-ischemic damage or mitochondrial dysfunction. However, there is no evidence to suggest our patient ever had any hypoxicischemic event. It is plausible that these MRI findings could be associated with some of her neurocognitive delays. She had a normal bone age study at age 10.5 years. Menarche occurred at 13 years of age and she has had metrorrhagia since then. At first, menses occurred every 2-3 months, lasting 10 days with approximately 4 days of heavy bleeding (needing to change pads every $1.5 \mathrm{hr}$ ). These symptoms have been successfully managed with ethinyl estradiol and levonorgestrel. At age 14.5 years, her head circumference was $58 \mathrm{~cm}$ (between +2.5 and $+3 \mathrm{SD}$ ), weight was $92 \mathrm{~kg}(+2.29 \mathrm{SD})$, and height was $171 \mathrm{~cm}$ $(+1.48 \mathrm{SD})$. The dysmorphology exam at this time was remarkable for macrocephaly, mild enophthalmos bilaterally, acanthosis nigricans affecting the neck and flexor creases of elbows, abdominal striae, and hirsutism of the face, neck, and abdomen. There were no other notable dysmorphic features (Figure 1).

\section{2 | Genetic testing}

Chromosome analysis and Prader-Willi/Angelman syndrome methylation tests were both normal. Chromosome microarray analysis identified numerous regions of copy neutral absence of single nucleotide polymorphism heterozygosity. WES identified a homozygous, likely pathogenic variant in the membrane bound $\mathrm{O}$-acyltransferase domain containing 7 (MBOAT7) gene (c.855-2A>G). Cosegregation analysis of 


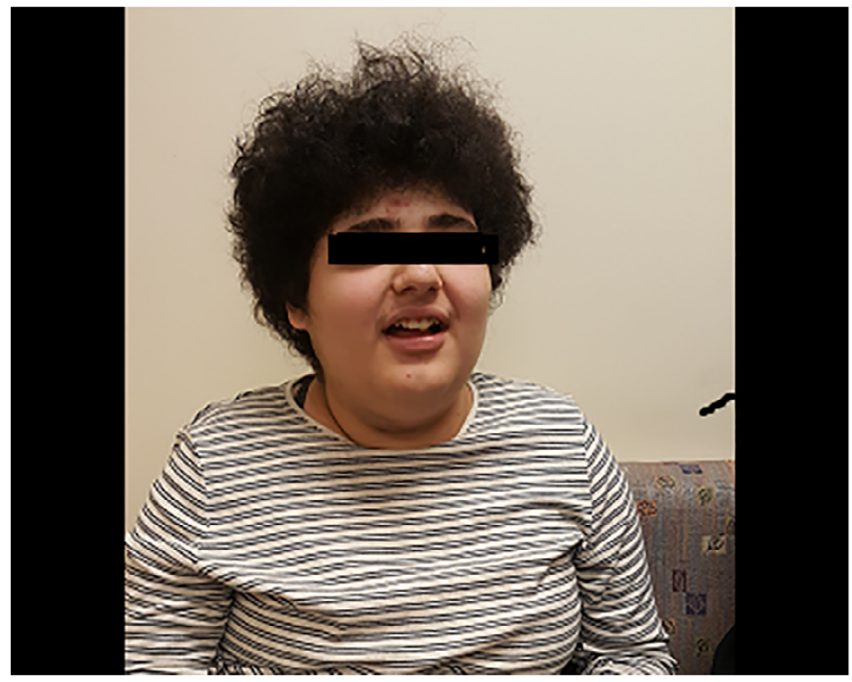

FIGURE 1 Frontal profile facial view of the proband at 14 years 11 months of age [Color figure can be viewed at wileyonlinelibrary.com]

the $c .855-2 A>G$ alteration revealed that the proband's unaffected mother and unaffected father were both heterozygous for the variant.

\section{3 | Psychiatric evaluation}

The patient was referred to psychiatry at 14 years of age for diagnosis and management of her behavioral symptoms including impulsivity, hyperactivity, defiance, perseverative behaviors, disruptiveness, and hyperphagia. These symptoms were pervasive across environments and caused distress to the parents and family. Psychological testing demonstrated a verbal and related behavior capacity in the range of 1-18 months of age. After initial comprehensive psychiatric evaluation her presentation was most consistent with that of ASD, unspecified anxiety disorder, and ADHD. There was no reported family history of intellectual disability, ASD, or other psychiatric diagnosis.

Developmental concerns were noted when she was unable to sit up independently and not crawling at age 9 months. She walked by age 2.5 years. Parents describe this patient as very rigid in having strict routines, needing to be repeated in exact ways, from the same people, in the same tone of voice, and struggling with transitions from a very young age. Regression in behaviors is often precipitated by changes in schedule. While parents deny a sustained restricted interest, she does enjoy playing with electronics (phones and computers), and is easily reinforced by food. She also has sensory avoidance of certain sounds including, fireworks and the "happy birthday" song. Social deficits were also noted at a very young age due to trouble with interpersonal skills. She is slow to warm up and often does not interact with others whom are not her mother, father, grandmother, or cousin. She is generally avoidant and suspicious of others; she prefers solitary play or with just her parents. Her eye contact is generally intermittent, rarely sustained, and often avoidant. Social reciprocity is lacking; she often will not follow commands and becomes perseverative on particular topics of interest to her. Along with delay in motor milestones, speech delays were noted around age 1.5 years when she was essentially nonverbal. At the age of 14 years, her vocabulary consists mostly of one to two word phrases, for example, "I done" or "mama." She expresses about 10 words in English. She does understand both English and Arabic with her receptive language being much more developed than expressive language. Finally, disruptive behaviors have been consistent, since she was young. These include yelling, spitting, shoving, hitting, crying, oppositionality, senseless refusal of requests, and throwing items. Such behaviors can be noted in the setting of limits, but also at times are unprovoked. She often becomes dysregulated if requests are not met immediately. She was evaluated through Michigan's Community Mental Health using the ADOS2 and ADI-R, both of which confirmed the diagnosis of ASD.

Comorbid with ASD was prominent symptoms of anxiety. She is wary of all new faces, very slow to warm up, easily tearful, and generally avoidant of new places and people. The family often has to allow for extra time for her to accustom herself to a new situation before she will enter. She often will stand outside the door of a doctor's office or require 10-25 min to get out of the car in a new environment. If not for her parents' insistence, her preference would be to stay in the home. She has a lot of trouble with meeting new people, and often will not go places where the door has to be shut. She currently sleeps well with pharmacological interventions; however, prior to initiation of medication, sleep was fragmented with $20+$ awakenings per night. She uses Clonidine $0.1 \mathrm{mg}$ at bedtime with good effect. The multidimensional anxiety scale for children was completed, which supported the diagnosis of unspecified anxiety disorder, with scores well in the clinically significant range.

Upon evaluation, ADHD was suspected due to descriptions of hyperactivity, inattention, and distractibility. Vanderbilt rating scale was significant for primarily inattention issues, however, on multiple mental status exams, it was noted that she could be intrusive, distractible, and hyperactive. Parents described her as active all day with difficulty sitting down to watch television, or playing with just one activity. She has a tendency to switch activities within a few minutes and requires frequent redirection. She paces around at home and it takes a significant amount of time to settle her to bed.

Food appeared to be a major reinforcer of behaviors. Her parents reported that she always thinks about food and generally overeats. She is above the 99th percentile for weight. Her parents keep locks on the refrigerator and cupboard doors. If not, she will forage as much food as she can until she is interrupted. Her parents describe a lack of understanding of satiety and she needs frequent redirection away from food. She often eats five to six times per day and would eat more if she were provided with the opportunity. Her symptoms cause much distress within the home. While many antipsychotics can increase appetite, this individual was never on a medication that would have this effect until August 2019. Her hyperphagia has remained unchanged despite the addition of Abilify $2 \mathrm{mg}$ at bedtime. While on Abilify, she has had no worsening behaviors. 


\section{4 | DISCUSSION}

In this report, we describe a female with a novel homozygous, likely pathogenic variant in the MBOAT7 gene (c.855-2A>G) identified by WES who has a history of global developmental delay, intellectual disability, overgrowth with macrocephaly, metrorrhagia, seizures, basal ganglia hyperintensities, nystagmus, strabismus with amblyopia, ASD, ADHD, anxiety, irritable mood, aggressive outbursts, and hyperphagia. She does not have a history of appendicular hypertonia nor cortical atrophy as described in previous patients (Johansen et al., 2016). The c.855-2A>G variant is not present in ExAC, ClinVar, 1,000 Genomes Project, or the Genome Aggregation Database (accessed July 2018). Variants within the canonical splice acceptor site are typically considered pathogenic (Richards et al., 2015). The nucleotide position affected in our patient is well conserved in the MBOAT7 homologs of available sequenced vertebrate species. In silico models (BDGP \& ESEfinder) predict this alteration to abolish the native splice acceptor site; however, functional evidence for this variant is unavailable. Studies have shown that LPIAT1, the protein encoded from MBOAT7, is essential for cortical lamination, which is necessary for normal neuronal development (Lee et al., 2012). MBOAT7 knockout mice have abnormal cortical lamination and delayed neuronal migration with atrophy of the cerebral cortex and hippocampus. These mice were also typically smaller than their littermates, had unsteady gaits, dysmorphic features, difficulty feeding, and early death (Anderson et al., 2013; Lee et al., 2012). Work with this mutant and other models will be helpful in identifying the genetic pathways affected in LPIAT1 deficiency.

Many of our patient's symptoms are consistent with others with MBOAT7-related intellectual disability first described in humans by Johansen et al. (2016). That report described 16 individuals, all born from consanguineous parents, who all have homozygous pathogenic variants in the MBOAT7 gene confirming their diagnosis of MBOAT7related intellectual disability. The most common symptoms observed in these individuals include: intellectual disability (16/16) with many being non-verbal with delayed motor milestones (walking typically achieved between 2-7 years) (9/16), seizures (10/16), truncal hypotonia in infancy (16/16), appendicular hypertonia (16/16), ASD (7/16), and, less commonly, brain MRI findings, such as cortical atrophy and polymicrogyria (2/16). Characterization of the psychiatric aspects of MBOAT7-related intellectual disability, other than ASD, have not been presented.

Psychiatric diagnoses are especially prevalent in individuals with intellectual disability (Dykens, 2000). As with our patient, a full psychiatric assessment would be beneficial to those who have MBOAT7-related intellectual disability. An ASD evaluation may provide both diagnostic clarification and treatment goals, which may help to alleviate symptoms and improve overall quality of life for both patients and families. Confirming other psychiatric diagnoses, including ASD, can have implications on school placement, burdens of care, and the lens through which many children are viewed. Often times, certain traditional options may not benefit these children, and appropriate treatment may begin only after psychiatric diagnoses are confirmed. We recommend that all patients with MBOAT7-related intellectual disability have a full psychiatric evaluation to rule in or out ASD and other comorbid psychopathology, as psychiatric interventions may provide alternate therapeutic interventions and improve symptoms severity.

As the use of whole exome and genome sequencing continues to rise, it is expected that more individuals with MBOAT7-related intellectual disability will be identified. Our patient's phenotype expands upon what has been previously reported revealing overgrowth with macrocephaly, metrorrhagia, ophthalmological abnormalities, basal ganglia hyperintensities, Unspecified Anxiety Disorder, ADHD, and hyperphagia without having appendicular hypertonia or cortical atrophy as additional characteristics. Additional older patients with this disorder need to be evaluated and published in order to delineate the full clinical spectrum of this rare syndrome. As this occurs, it will contribute to the development of specific medical management guidelines.

\section{ACKNOWLEDGMENTS}

The authors would like to thank the patient and her family for their contribution. Dr. Innis acknowledges support from the Morton S. and Henrietta K. Sellner Professorship in Human Genetics.

\section{CONFLICT OF INTEREST}

The authors report no conflict of interest.

\section{ORCID}

Joseph E. Jacher (D) https://orcid.org/0000-0002-0725-2871

\section{REFERENCES}

Anderson, K. E., Kielkowska, A., Durrant, T. N., Juvin, V., Clark, J., Stephens, L. R., \& Hawkins, P. T. (2013). Lysophosphatidylinositolacyltransferase-1 (LPIAT1) is required to maintain physiological levels of Ptdlns and Ptdlns $\mathrm{P}_{2}$ in the mouse. PLoS One, 8(3), e58425. http:// doi.org/10.1371/journal.pone.0058425

Clark, M. M., Stark, Z., Farnaes, L., Tan, T. Y., White, S. M., Dimmock, D., \& Kingsmore, S. F. (2018). Meta-analysis of the diagnostic and clinical utility of genome and exome sequencing and chromosomal microarray in children with suspected genetic diseases. NPJ Genomic Medicine, 3 (16), 16. http://doi.org/10.1038/s41525-018-0053-8

Dykens, E. (2000). Annotation: Psychopathology in children with intellectual disability. The Journal of Child Psychology and Psychiatry and Allied Disciplines, 41(4), 407-417.

Emerson, E. (2003). Prevalence of psychiatric disorders in children and adolescents with and without intellectual disability. Journal of Intellectual Disability Research, 47(1), 51-58.

Johansen, A., Rosti, R. O., Musaev, D., Sticca, E., Harripaul, R., Zaki, M., ... Abou Jamra, R. (2016). Mutations in MBOAT7, encoding lysophosphatidylinositol acyltransferase I, lead to intellectual disability accompanied by epilepsy and autistic features. American Journal of Human Genetics, 99(4), 912-916.

Lee, H. C., Inoue, T., Sasaki, J., Kubo, T., Matsuda, S., Nakasaki, Y., ... Arai, H. (2012). LPIAT1 regulates arachidonic acid content in 
phosphatidylinositol and is required for cortical lamination in mice. Molecular Biology of the Cell, 23(24), 4689-4700.

Nazeer, A., \& Ghaziuddin, M. (2012). Autism spectrum disorders: Clinical features and diagnosis. Pediatric Clinics of North America, 59(1), 19-25. https://doi.org/10.1016/j.pcl.2011.10.007

Richards, S., Aziz, N., Bale, S., Bick, D., Das, S., Gastier-Foster, J., ... ACMG Laboratory Quality Assurance Committee. (2015). Standards and guidelines for the interpretation of sequence variants: A joint consensus recommendation of the American College of Medical Genetics and Genomics and the Association for Molecular Pathology. Genetics in Medicine: Official Journal of the American College of Medical Genetics, 17(5), 405-424.
The Deciphering Developmental Disorders Study. (2017). Prevalence and architecture of de novo mutations in developmental disorders. Nature, 542(7642), 433-438. http://doi.org/10.1038/nature21062

How to cite this article: Jacher JE, Roy N, Ghaziuddin M, Innis JW. Expanding the phenotypic spectrum of MBOAT7related intellectual disability. Am J Med Genet Part B. 2019; 180B:483-487. https://doi.org/10.1002/ajmg.b.32749 